\title{
Die Aussagekraft vorzeitlicher Bodenbildungen als Klima- und Zeitindices
}

\author{
Von OtMar Seuffert, Würzburg
}

$\mathrm{Z}$ us a mmen $\mathrm{f}$ as sung. Die Aussagekraft von Fossil- und Reliktböden als Indikatoren vorzeitlicher Klimate oder Zeiteinheiten ist sehr unterschiedlich. Sie wird als recht ansehnlich erachtet bei der Erfassung früherer Klimate, von denen häufig der allgemeine Typus und teilweise auch das ungefähre Temperatur- und Niederschlagsvolumen ermittelt werden können. Dagegen bleiben zuverlässige Angaben über die Bildungsdauer fossiler Böden weitgehend auf Relativwerte beschränkt, die zudem nur unter sehr günstigen Voraussetzungen überhaupt erstellbar sind. Absolute Zeitabschätzungen sind stets grobe Näherungswerte.

Diese Ergebnisse sind jedoch nur dann von Wert, wenn der Entwicklungsstand der in Frage stehenden Vorzeitböden zuverlässig fes gelegt werden kann, denn über alle Gegegbenheiten hinaus, die das Maß der Aussagen beeinflussen, gilt der Grundsatz, daß Vorzeitklimate nur aus Klimaxböden erschlossen werden können, während umgekehrt eine Abschätzung der Bildungsdauer allein aus unreifen Böden möglich ist.

$\mathrm{Sum} \mathrm{mary}$. The value of fossil and relict soils as indicators of past climates or periods differs greatly. It is rather high when we want to reconstruct former climates, of which we can often find out the general type and partly also the approximate amount of temperature and precipitation. On the other hand reliable statements on the time which fossil soils take for their development are largely confined to relative data, which we can only get under very favourable circumstances. Absolute estimates of time can always be done only approximately.

These results, however, are only useful, if the state of development of the fossil soils under discussion can be determined with sufficient reliability. Beyond all factors which influence the exactness of our statements we must keep in mind the principle, that fossil climates can only be reconstructed out of climax soils, whereas the estimation of the duration of soil development is only possible with immature soils.

Unter dem ganzen Bündel von Hilfsmitteln, die zur Erforschung der Umweltbedingungen vergangener Perioden der Erdgeschichte herangezogen werden, spielen die Relikt- und Fossilböden eine große Rolle.

Vor allem in der Eiszeitforschung stellt die Auswertung solcher Böden ein viel gebräuchliches Indiz dar, das von nahezu allen beteiligten Disziplinen (Geologie, Geographie, Bodenkunde, Biologie u. a.) verwendet wird. Besondere Bedeutung haben hierbei die fossilen Lößböden erlangt, deren Bearbeitung im Rahmen der "Lößstratigraphie“ heute als das wohl wichtigste oder zumindest als das verbreitetste Hilfsmittel bei der Erschließung der Quartärgeschichte gelten kann.

Allein diese weitverbreitete Untersuchung von Vorzeitböden sollte nun eigentlich auch die Gewähr dafür geben, daß das Maß und der Spielraum der Aussagen, die darauf beruhen, klar erkannt wird.

Dies aber ist u. E. nicht immer der Fall, da gerade in jüngster Zeit einige Schlußfolgerungen, die auf dieser Methode aufbauen, merklich über das Ziel, d. h. über die Aussagemöglichkeiten, hinausgehen.

Darauf hinzuweisen und einige Gedanken zu der damit verbundenen Problematik zu äußern, soll der Zweck dieses kleinen Beitrags sein.

\section{Vorzeitböden als Klimaindices}

Die wissenschaftliche Ausdeutung von Fossil- oder Reliktböden geht von der Erkenntnis aus, daß jeder Boden ein Verwitterungsprodukt ist, das aus einem an der Erdoberfläche gelegenen Ausgangsmaterial (i. d. Regel das Gestein oder ein vorgebildeter Boden) unter dem gemeinsamen Einfluß von Klima, Lebewelt und Relief einen Entwicklungsprozeß durchmacht, der daraufhinzielt, ein möglichst vollkommenes dynamisches Gleichgewicht zwischen Ursachen (d. s. die beteiligten bodenbildenden Faktoren) und Wirkung (d. i. der Boden) zu erreichen bzw. zu bewahren. 
Es ist seit langem bekannt, daß dem Klima bei diesem Geschehen eine übergeordnete Bedeutung zukommt. Es bestimmt überall auf der Erde die großräumige Bodenzonierung (sog. zonale Böden). Alle übrigen Einflußmomente (Gestein, Lebewelt, Relief u. a.) bewirken normalerweise nur sekundäre Veränderungen der Bodenbildung innerhalb dieses übergeordneten Klimarahmens. Sie können höchstens lokal und in seltenen Ausnahmefällen auch regional zum bestimmenden Gestaltungselement der Bodenentwicklung werden (sog. intrazonale und azonale Böden).

Sichtbarer Ausdruck und Ergebnis dieser Vorgänge ist das Bodenprofil. Es ist gewissermaßen der „Katalog“, in dem das Ursachengefüge des jeweiligen Bodens in mehr oder weniger verschlüsselter Form aufgezeichnet und demzufolge erschließbar ist.

Diese Erfassung der Wirkungsfaktoren geschieht bei den rezenten Böden durch die unmittelbare Korrelation der Bodenkriterien mit den heutigen Umweltverhältnissen. Die diesbezügliche Auswertung der Vorzeitböden (Fossil- und Reliktböden) erfolgt demgegenüber durch einen Vergleich mit entsprechenden rezenten Böden bzw. Bodenteilen, deren Wirkungsgefüge bekannt ist. Hierbei kann entweder eine makroskopische Gegenüberstellung erfolgen oder es werden Einzelerscheinungen vorzeitlicher Böden aufgrund ihrer Eigenart ganz bestimmten heutigen Bodenbildungsprozessen zugeordnet.

Diese Möglichkeiten sind nicht neu. Sie sind vielmehr häufig erprobt und haben ihre Bewährungsprobe längst bestanden. Trotzdem soll hier gerade auf die Schwierigkeiten und Unzulänglichkeiten hingewiesen werden, die mit ihnen verbunden sind. Diese allein vermögen schließlich den Umfang und den Zuverlässigkeitsgrad ihrer Aussage zu umreißen.

Die ersten Schwierigkeiten, die der Ausdeutung von Vorzeitböden als Klimaindices entgegenstehen, sind in der unterschiedlichen Aussagekraft des Vergleichsmaßstabes, also des heutigen Bodens begründet, sei es, daß der rezente Boden nur eine sehr vage Klimaaussage zuläßt (insbesondere bei den sog. azonalen und intrazonalen Böden), oder sei es, daß seine „Klimaträchtigkeit“ noch gar nicht genau genug eingeschätzt werden kann. Es ist nämlich durchaus nicht so, daß alle heutigen Böden so gut bekannt und abgeklärt wären, als daß man in jedem Fall wirklich detailierte Aussagen über ihren Bildungsmechanismus zu machen vermöchte. Außerdem haben viele Böden, die genetisch durchaus klassifizierbar sind und auf ihren klimatischen Aussagewert hin eingeordnet werden können, einen recht beträchtlichen Klimaspielraum, so daß gar nicht erwartet werden kann, daß entsprechende Vorzeitböden, die zudem häufig nur annähernd vergleichbar sind, eine genaue Klimaaussage ermöglichen.

Ein zweites Hindernis, das einer umfassenden Klimazeugenschaft, wie überhaupt einer sehr in Einzelheiten gehenden Ausdeutung von Vorzeitböden entgegenwirkt, bilden die Ungenauigkeiten, die zwangsläufig bei der Parallelisierung so komplizierter Gebilde wie der Böden auftreten. Es handelt sich dabei ja nicht um einen simplen mathematischen Vergleich festliegender Daten, sondern um die kritisch abwägende Gegenüberstellung vielfältiger dynamischer Vorgänge der Bodenbildung, die innerhalb der periphersten Bereiche der Erdoberfläche zu bestimmten Veränderungen des Ausgangsmaterials führen und aus diesen erschlossen werden können.

Zwar sind viele dieser Prozesse im Wesen und in der Richtung ihres Ablaufs durchaus klimaspezifisch, wie etwa die Vorgänge der Podsolierung, der Serosemierung, der Rubefizierung u. a., ihr Ausmaß und ihre Geschwindigkeit aber sind nur in den wenigsten Fällen eine einfache Proportion der Klimadaten. Sie werden vielmehr von einem vielschichtigen Ursachengefüge gesteuert, in dem zwar das Klima eine meist überragende Rolle spielt, aber es ist eben doch nur ein Faktor neben einer Reihe anderer, von denen jeder für sich allein oder mehrere im gegenseitigen Zusammenwirken durchaus Veränderungen beträchtlicher Größenordnung bewirken können. 
Um dieses Wirkungsgefüge jedoch in seine einzelnen Elemente aufzugliedern, dazu fehlen uns häufig nicht nur hinreichende Kenntnisse über den genauen Ablauf der Vorgänge und über die darin enthaltene gegenseitige Abhängigkeit der einzelnen Faktoren, sondern häufig auch die technischen Voraussetzungen.

Zwar ist man heute dank moderner Laboratoriumsmethoden (z. B. mikromorphologische Untersuchungen nach W. L. KuBIENA; Farbremissionsmessungen; röntgengeographische, differentialthermoanalytische und elektronenmikroskopische Tonmineralbestimmung u. a.) schon in der Lage, eine Reihe von Bodenentwicklungsprozessen bzw. die mit ihnen verbundenen Veränderungen des Ausgangsmaterials zu analysieren und kann auf diese Weise manchen Boden in seine Einzelvorgänge bzw. Einzelbestandteile zerlegen. Jeder Vergleich zweier oder mehrerer Böden aber ist letzten Endes noch immer nichts anderes, als eine gegenseitige Abwägung von Tendenzen, deren quantitative Beziehungen allenfalls durch die grobe Parallelisierung von Verhältniszahlen verdeutlicht und anhand einer möglichst großen Zahl von Vergleichspartnern wahrscheinlich gemacht werden können.

Ein wirklich exakter, quantitativer Vergleich zweier oder mehrerer Böden bzw. der in ihnen ablaufenden Bodenbildungsprozesse bleibt uns hingegen bis heute verwehrt. Dazu fehlt uns ganz einfach jeglicher Standardmaßstab, der für die zu vergleichenden Individuen eine echte Konstante darstellen würde.

Ein drittes Erschwernis einer genaueren Klimacharakterisierung von Vorzeitböden stellt deren mangelhafte Erhaltung dar. Viele sind nämlich durch spätere Abtragung geköptt oder sonstwie verstümmelt oder sie wurden durch postbildungszeitliche Umlagerungen oder Uberprägungen in ihrer Struktur und in ihrer inneren Gliederung verändert. Dies mag man schon daraus ersehen, daß die überwiegende Zahl der Vorzeitböden zur Gruppe der Reliktböden gehört, während echte Fossilböden nur selten erhalten sind.

Der an sich ideale Ausgangspunkt eines Vergleichs zwischen vorzeitlicher und heutiger Bodenbildung, nämlich ein vollständig originäres Fossilbodenprofil, ist also nur in Ausnahmefällen verwirklicht. Er ist bei uns weitgehend auf solche quartären Lößböden beschränkt, bei denen eine jüngere Überwehung so schnell erfolgte und eine so große Mächtigkeit erreichte, daß die synsedimentäre Bodenbildung damit weder Schritt halten konnte, noch das begrabene Bodenprofil vom Tiefgang einer späteren Bodenbildung erreicht wurde.

Selbst dabei bleibt jedoch ein kleiner Unsicherheitsfaktor, da die Frage, inwieweit der verschïttete bzw. überwehte Boden schon Zeichen der Umstände in sich trägt, die zu seiner Fossilisierung führten, nicht immer ohne weiteres beantwortet werden kann.

In den Fällen jedoch, in denen die Vorzeitböden nicht mit ihrer ursprünglichen Struktur oder nur als anerodierte Reste erhalten sind, wird die Vergleichsrechnung durch die schon vor der eigentlichen Gegenüberstellung notwendige Rekonstruktion der originären Ausgangsbasis mit einem zusätzlichen Fehlerglied versehen.

Wir ersehen aus all dem, daß die Erschließung fossiler Klimafaktoren auf der Basis vorzeitlicher Bodenbildungen durch eine Reihe potentieller und zwangsläufiger Fehlerquellen behindert wird. Diese reichen von der schlechten Erhaltung vieler Vorzeitböden über die Unzulänglichkeiten der Vergleichsverfahren bis zur mangelnden Klimacharakterisierung mancher rezenter Böden.

Das heißt freilich nicht, daß die Vorzeitböden gänzlich bedeutungslos wären für die Erhellung der Klimageschichte. Bei entsprechend sorgfältiger Abwägung aller Begleitumstände erscheint es vielmehr durchaus möglich, zumindest den Klimatypus, der zu ihrer Bildung führte oder für den Ablauf bestimmter Vorgänge verantwortlich war, zu erkennen.

Fossil- und Reliktböden sowie z. T. auch einzelne pedologische Prozesse bzw. Strukturen können also etwa dazu verwendet werden, um den allgemeinen Typus des beteiligten 
Klimas, d.h. die Tendenzen im Jahresgang von Temperatur und Feuchtigkeit, sowie u. U. auch das Temperatur- und Niederschlagsvolumen innerhalb begrenzter Größenordnungen aufzuklären. Dies ist mit durchaus beachtlichem Erfolg beispielsweise für die quartäre Klimaabfolge geschehen. Daneben ist es im Bereich der lößbedeckten Gebiete Europas auf diesem Wege sogar gelungen, das räumliche Nebeneinander fossiler Klimate näher zu umreißen, wozu vor allem K. BrunNacker (1956, 1957, 1959 u. 1964), J. FINK (1956, 1961, 1964 u. 1965), sowie W. L. Kubiena (1954 u. 1956) wertvolle Beiträge geliefert haben.

Eine wirklich zuverlässige, quantitative Festlegung einzelner Klimaelemente, also etwa eine auf Grad oder gar Zehntelgrad genaue Temperaturangabe (vgl. z. B. K. SchäDEL und J. Werner, 1963, S. 14/15), eine entsprechend detailierte Erkenntnis des fossilen Niederschlagsvolumens oder aber eine ähnlich genaue quantitative Einstufung der jeweiligen jahreszeitlichen Temperatur/Feuchte-Relation können wir allein mit dieser Methode nicht erwarten. Wir können dies deshalb nicht, weil derartige quantitative Ergebnisse nur von einer adäquaten quantitativen Methode erbracht werden könnten, und um eine solche handelt es sich hierbei zweifellos nicht.

Unter diesen Aspekten wird schließlich auch verständlich, daß diese Methode bisher immer dann nicht ausreichte, um zu einem klaren Bild der Vorzeitklimate zu gelangen, wenn die in Frage stehenden Fossilböden das Produkt untergeordneter Klimaschwankungen waren. Dies betrifft beispielsweise die sog. Innerwürmböden, deren klimatische Stellung nach wie vor heftig umstritten ist, wobei eine reine Feuchteoszillation als Bildungsursache genau so in Erwägung gezogen wird, wie eine deutliche, fast interglaziale Ausmaße erreichende Temperatur- und Niederschlagsschwankung. Wenn wir bedenken, daß die außerordentlich unterschiedliche Deutung dieser Gebilde möglicherweise weniger auf einer wirklich so weitgespannten $\mathrm{K}$ ! imaabstufung beruht, als vielmehr auf einer teilweisen klimatischen Überbewertung örtlicher Ausbildungsunterschiede aufgrund einer Überforderung der Arbeitsmethode, dann werden diese widersprüchlichen Ergebnisse vielleicht eher verständlich.

In jedem Fall bestätigt die Erfolgsbilanz unsere theoretische Erkenntnis, daß die Ausdeutung von Vorzeitböden zwar eine ausgezeichnete Methode zur Erkenntnis qualitativer Klimagegebenheiten der Vorzeit darstellt, daß jedoch die Erschließung von Details fossiler Klimaelemente keine zuverlässigen Ergebnisse zu liefern vermag.

Selbst eine solche allgemeine Charakterisierung vorzeitlicher Klimaelemente aufgrund der Auswertung von Fossil- und Reliktböden beruht jedoch noch auf einer wesentlichen Voraussetzung, nämlich darauf, daß der auszuwertende Boden vollkommen oder doch weitestgehend an seine Bildungsbedingungen angepaßt ist. Nur ein Gebilde, das seinem Ursachengefüge bis in alle Einzelheiten entspricht, kann schließlich dazu dienen, dieses auch mit ausreichender Sicherheit zu identifizieren.

Ein unreifer Boden ist hingegen meist ungeeignet, um auf ihm eine wirklich qualifizierte Aussage über das zu seiner Bildung notwendige Faktorengefüge und damit auch über das Klima zu ermöglichen. Zwar gibt es eine Reihe pedologischer Prozesse, deren Verlauf auch bei einem noch nicht ausgereiften Boden schon wertvolle Angaben über die klimatischen Bildungsumstände ermöglicht, doch das Maß der Aussagemöglichkeiten und deren Zuverlässigkeit steigt in jedem Fall mit zunehmender Bodenreife und erreicht sein Maximum dann, wenn der Boden sich im Klimax-Stadium befindet.

Ehe nun aber ein Boden diesen Reifezustand erreicht, braucht er eine ausreichende Zeit, die in Abhängigkeit von der jeweiligen Verwitterungs- bzw. Bodenbildungsintensität und damit vor allem vom Klima innerhalb recht ansehnlicher Zeitintervalle schwanken kann und im Mittel etwa $10^{3}$ bis $10^{5}$ Jahre betragen dürfte (vgl. auch J. BüDEL, 1963).

Wir stehen damit vor der Notwendigkeit, alle vorzeitlichen Böden, die den Klimaxzustand nicht erreicht haben, als Klimaindices auszuklammern. 
Das ist eine klare Forderung. Ihre Erfüllung aber ist häufig gar nicht oder doch nur sehr unvollkommen gewährleistet. Ob nämlich ein Klimaxboden vorliegt oder nicht, das läßt sich vielfach gar nicht oder nur mit mehr oder weniger großen Einschränkungen aus dem Profil ablesen. Zwar können uns Struktur, Mächtigkeit und innere Gliederung eines Bodens einen Hinweis auf den Verwitterungsfortschritt des Ausgangsmaterials geben, ein Beweis für das Vorhandensein eines Klimaxbodens aber ist das nicht. Genau so nämlich, wie sich einerseits ein recht schwach entwickeltes und wenig differenziertes Bodenprofil im Stadium der Reife befinden kann, muß umgekehrt ein gut ausgeprägter, mächtiger und deutlich horizontierter Boden nicht zwangsläufig auch ein reifer Boden sein. Viele Böden können vielmehr - theoretisch gesehen - sowohl eine Rohform, als auch ein Klimaxboden sein.

Eine wirklich zuverlässige Trennung in reife und unreife Böden ist deshalb außerordentlich schwierig und oft mit pedologischen Indizien allein nicht durchführbar.

Die Verwendung von Vorzeitböden als Klimaindices ist also - das bleibt das endgültige Fazit unserer Betrachtungen zu dieser Frage - von einer gesicherten Erkenntnis der Gleichgewichtslage bzw. des Bodenreifezustandes abhängig. Erst wenn dieser zuverlässig festgelegt werden kann, was u. U. aus dem Aufbau des Profils wahrscheinlich gemacht werden kann, was vielfach aber erst unter Zuhilfenahme anderer, nichtpedologischer (geologischer, paläogeographischer, paläobotanischer u. a.) Indizien möglich ist, vermögen Fossil- und Reliktböden als beweiskräftige Anzeiger vorzeitlicher Klimacharaktere herangezogen zu werden.

\section{Vorzeitböden als Zeitindices}

Ein Problem, das erst in jüngster Zeit Gegenstand intensiverer wissenschaftlicher Betrachtungen wurde, ist die Frage nach der Bildungsdauer vorzeitlicher Böden. Anlaß dieser Untersuchungen ist die Überlegung, $d a ß$ auf diese Weise bei einigermaßen verläßlichen Ergebnissen eine Altersabschätzung einzelner Klimaphasen der Vorzeit bzw. bei entsprechend lückenloser Summierung gar eine Festlegung der Dauer größerer geologischer Zeiträume, wie etwa des Gesamtquartärs, möglich sein könnte (vgl. K. BRUnNACKER, 1965, S. 415 ff.).

Wenn wir uns vor Augen halten, daß sowohl die Entstehung eines Bodens als Ganzes, wie auch jeder einzelne der dabei ablaufenden chemisch-physikalischen Prozesse nicht nur eine Folge klimatischer, geologischer, biologischer und morphologischer Einflußelemente darstellt, sondern genau so als eine Funktion der Zeit betrachtet werden muß, dann erscheint uns die Darstellung dieses Zeitfaktors aufgrund der Auswertung des Bodenentwicklungszustandes sehr wohl möglich.

Als Ausgangspunkt und Bemessungsgrundlage für eine derartige Untersuchung kann sowohl der Fortschritt einzelner Bodenbildungsprozesse, als auch der Entwicklungszustand des Gesamtbodens dienen.

In praxi wird allerdings eine Abschätzung der Bodenbildungsdauer aufgrund des Entwicklungszustandes bestimmter pedogenetischer Vorgänge kaum verwirklicht, da hierfür die notwendigen Grundlagen und Kenntnisse über die zeitlichen Beziehungen der einzelnen Prozesse noch zu wenig bekannt sind. Immerhin erscheint dieser Erkenntnisweg der Beachtung im Rahmen der weiteren Entwicklung bodenkundlicher Forschung und damit der Erwähnung wert zu sein.

Die nahezu alleinige Basis für „Zeitdiagnosen“ bildet also der jeweilige Grad der Gesamtbodenentwicklung, der sowohl im Profilaufbau, als auch in der Bodenmächtigkeit zum Ausdruck kommt.

Hiervon ist die Bodendicke sowohl am einfachsten zu handhaben, als auch am ehesten feststellbar, während die „innere Gliederung" eines Bodens zu viele unterschiedliche Aspekte hat, als daß eine darauf fußende Alterseinschätzung bzw. ein Vergleich heute schon fruchtbare Ergebnisse erwarten ließe. 
Trotz der leichten Meßbarkeit der Bodendicke ist jedoch die Erschließung der Bildungsdauer vorzeitlicher Böden auf dieser Grundlage mit einer Reihe von Schwierigkeiten und Fehlerquellen behaftet, die genau so, wie dies bei der Ermittlung vorzeitlicher Klimata der Fall war, in der beschränkten Aussagefähigkeit der heutigen Vergleichspartner, in den Unzulänglichkeiten der Parallelisierung und schließlich auch in der lückenhaften Erhaltung von Fossilböden zu suchen sind.

Hierbei ist vor allem der Aussagewert der heutigen Böden ein ganz besonderes Problem, denn unter allen Determinismen, die bei der Entstehung rezenter Böden eine Rolle spielen, ist die Zeit/Dicke-Korrelation sicherlich am schwersten einzustufen. Dies liegt daran, daß die Bodenmächtigkeit das Produkt einer Reihe von Faktoren ist, unter denen der Zeit keineswegs immer die überragende Rolle zukommt. Vielmehr spielen hierbei fast immer das Klima und die Morphodynamik und manchmal auch das Gestein oder gar die Vegetation eine ausschlaggebende Rolle. Dies läßt sich schon daraus erschließen, daß in der heute auf der Erdoberfläche sichtbaren Verteilung der Bodenmächtigkeiten weniger die - allerdings schwer überschaubare - zeitliche Komponente, als vielmehr vor allem klimabedingte Unterschiede projiziert sind, die eine deutlich zonale, d. h. breitenkreisparallele Ausrichtung anzeigen.

Wir ersehen daraus, daß ein Vergleich von Bodenmächtigkeiten mit dem Ziel einer Erfassung ihrer Bildungsdauer überhaupt nur dann Aussicht auf Erfolg besitzen kann, wenn das Alter der betreffenden Böden das einzige ist, in dem sie sich voneinander unterscheiden; wenn also das Wirkungsgefüge der $\mathrm{zu}$ vergleichenden Böden mit Ausnahme eben des Zeitfaktors praktisch identisch ist.

Bei derartig vergleichsfähigen Böden - aber auch nur dort - ist die Bodenmächtigkeit u. U. eine unmittelbare Funktion des Bodenalters, d. h. je größer die Bodendicke, um so älter ist der Boden und umgekehrt. Bei einer Gegenüberstellung zweier oder mehrerer solcher in ihrer Mächtigkeit gestaffelter Böden umfaßt folglich der mächtigste den längsten Bildungszeitraum, während die übrigen entsprechend ihrer Dickenrelation altersmäßig abgestuft sind.

Es liegt auf der Hand, daß derartig abgeleitete Altersunterschiede normalerweise nur relativen Charakter haben, doch kann man in manchen Fällen aufgrund der Erfahrungswerte bei rezenten Böden auch Rückschlüsse auf die absoluten Unterschiede in der Bildungsdauer ziehen, deren Genauigkeit allerdings wegen der sehr unsicheren Grundlage innerhalb weiter Grenzen schwanken kann.

Unter Zuhilfenahme dieser Methode hat etwa K. Brunnacker (s. o.) eine Altersabschätzung des Gesamtquartärs versucht, in der er die warmzeitlichen Perioden dieser geologischen Epoche aufgrund der in ihnen jeweils auftretenden mittleren Bodendicken in absolute Zeiteinheiten umwertet und diese schließlich mit den auf andere Weise gewonnenen Werten für die Kaltzeiten zu einem zeitlichen Gesamtbild des Quartärs vereinigt.

Diese, wie auch andere Arbeiten lassen erkennen, daß der Methode als solcher - abgesehen vielleicht von Vorbehalten, die den Grad der Genauigkeit betreffen - durchaus ein Aussagewert zuerkannt wird. Die Verwendbarkeit der Bodendicke als Anzeiger der Bodenbildungszeit scheint also unter den genannten Voraussetzungen gesichert.

Wir erachten es trotzdem für notwendig, dieses Indiz noch einmal mit einer ganz bestimmten Blickrichtung auf seinen Gültigkeitsumfang hin kritisch zu durchleuchten. Es fällt nämlich auf, daß in dieser Methode ein praktisch unbegrenzt andauernder Dickenzuwachs vorausgesetzt wird, d. h. die Bodenmächtigkeit wird bei gleichbleibenden sonstigen Bildungsbedingungen als eine ausschließliche Funktion der Zeit angesehen.

Wir sind demgegenüber der Meinung, daß die Bodendicke auch bei langzeitig gleichbleibendem Wirkungsgefüge nur solange eine Proportionale der Zeit ist, solange der Boden noch nicht das Stadium der Reife (Bodenklimax) erreicht hat. In dem Moment jedoch, in dem er in allen seinen Zügen den herrschenden Umweltbedingungen angepaßt ist, hat 
u. E. auch sein Dickenwachstum ein Ende gefunden. Von diesem Zeitpunkt an wird selbstverständlich immer unter der Voraussetzung einer gleichbleibenden Faktorenkonstellation - die Bodendicke nur noch bewahrt, aber nicht mehr weiter vermehrt.

Die Gründe dafür sind folgende: Jede Bodenbildung verläuft in Richtung auf ein labil allen Einflußfaktoren angepaßtes, dynamisches Gleichgewicht bzw. versucht dieses - wenn es einmal erreicht ist - zu erhalten. Diese Entwicklung vollzieht sich aber nicht nur in Form chemisch-physikalischer Umwandlungen, die im Innern des Ausgangsmaterials einem solchen Gleichgewichtszustand zustreben, sie zielt vielmehr genau so auf die Herstellung einer ausgewogenen äußeren Balance zwischen der Bodenbildung (d.h. dem Verwitterungsfortschritt) einerseits und der Reliefentwicklung (d.h. der Abtragung oder Ablagerung) andererseits.

Jeder Boden wird folglich im Stadium des Gleichgewichts nicht nur seine größtmögliche innere Umwandlung und Gliederung besitzen, sondern auch seine maximale Mächtigkeit.

Beides wird sich in der Folgezeit bei gleichbleibendem Ursachengefüge nicht mehr merklich verändern, da der Boden von diesem Zeitpunkt an unter Wahrung seines inneren Aufbaus stets genau so schnell in die Tiefe oder Höhe wächst, wie Material an seinem Dach ab- oder aufgetragen wird.

Für unsere Fragestellung bedeutet das, daß die Bodenmächtigkeit als Zeitindex nur dann überhaupt von Wert sein kann, wenn für die Vergleichspartner sowohl das Vorhandensein eines mit Ausnahme der Zeit weitgehend identischen Wirkungsgefüges (- vor allem ein gleiches oder sehr ähnliches Klima -), als auch die Nichtexistenz eines Klimaxstadiums gesichert ist.

$\mathrm{Da}$ wir annehmen dürfen, daß diese Voraussetzungen auch bei der Untersuchung von K. Brunnacker (s. o.) entweder nicht vollkommen ( - beim Klima -) oder aber gar nicht (- Nichtklimaxboden -) erfüllt waren, können wir uns auch seiner Beweisführung nicht vollinhaltlich anschließen. Das heißt allerdings nicht, daß wir die Ergebnisse seiner Schätzung ablehnen. Diese mag sowohl für die einzelnen Abschnitte, wie auch in ihrer Gesamtheit den angenommenen Größenordnungen entsprechen. Nur beweisen läßt sich dies u. E. mit Hilfe der Bodendicken nicht, zumindest nicht in diesem speziellen Fall.

\section{Literaturaus w a h 1 :}

BRUNNACKER, K.: Regionale Bodendifferenzierungen während der Würmeiszeit. Eiszeitalt. u. Gegenw., 7, 43-48, 1956. - - Die Geschichte der Böden im jüngeren Pleistozän in Bayern. Geol. Bavar., 34, München 1957. - - Zur Parallelisierung des Jungpleistozäns in den Periglazialgebieten Bayerns und seiner östlichen Nachbarländer. Geol. Jahrb., 76, 1959. - - Die Würmeiszeit im Lichte der Lößforschung. Rep. VIth Int. Congr. Quatern., Warschau 1961, 441-449, Lodz 1964. - - Schätzungen über die Dauer des Quartärs, insbesondere auf der Grundlage seiner Paläoböden. Geol. Rdsch., 54, 415-428, 1965.

BüDEL, J.: Klima-genetische Geomorphologie. Geogr. Rdsch. H. 7, 269-286, Juli 1963.

Fink, J.: Zur Korrelation der Terrassen und Lösse in Österreich. Eiszeitalt. u. Gegenw., 7, 49-77, 1956. - - Leitlinien einer österreichischen Quartärstratigraphie. Mitt. Geol. Ges. Wien, 53, 1961. - - Die Gliederung der Würmeiszeit in Ớsterreich. Rep. VIth Int. Congr. Quatern., Warschau 1961, 451-462, Lodz 1964. - - The Pleistocene in Eastern Austria. Geol. Soc. Amer., Spec. Paper 84, 179-199, 1965.

Kubiena, W. L.: Sobre el metodo de la paleoedafologia. Ann. Edafologia y Fisiologia vegetal, 13, 523-543, 1954. - - Zur Mikromorphologie, Systematik und Entwicklung der rezenten und fossilen Lößböden. Eiszeitalt. u. Gegenw., 7, 102-112, 1956. - - Prinzipien und Methodik der paläopedologischen Forschung im Dienste der Stratigraphie. Z. dt. geol. Ges., 111, 643-652, 1959. - - Paleosoils as indicators of paleoclimates. Unesco/NS/AZ. Symposium, Rome 1961.

SchädEL, K. \& Werner, J.: Neue Gesichtspunkte zur Stratigraphie des mittleren und älteren Pleistozäns im Rheingletschergebiet. Eiszeitalt. u. Gegenw., 14, 5-26, 1963.

Manuskr. eingeg. 18. 4. 1967.

Anschrift des Verf.: Dr. Otmar Seuffert, 8702 Zell b. Würzburg, Adalbert-Stifter-Weg 92. 